\title{
ON CERTAIN INVARIANTS OF TWO TRIANGLES*
}

\author{
BY \\ JOHN GALE HUN
}

The purposes of this paper are to express some of the simpler invariant relations of two triangles in terms of three fundamental invariants, and to consider the case of two triangles apolar both ways. The fundamental invariants are derived by a method first presented by HiLbert in a letter to Hermite. $\dagger$

\section{§1. The three fundamental invariants.}

With two plane curves, one of $n$th order $\alpha_{x}^{n}=0$ and the other of $n$th class $a_{\xi}^{n}=0$, there is associated the connex

$$
\alpha_{\nu} a_{\eta} \alpha_{a}^{n-1}=0,
$$

found by acting on the line curve with the polar of the point $y$ as to the point curve. $\ddagger$ The fixed points of this connex are given by the equations

which are consistent if

$$
a_{i} \alpha_{y} \alpha_{a}^{n-1}=\lambda y_{i}
$$

$$
\Delta(\lambda) \equiv\left|\begin{array}{lll}
a_{1} \alpha_{1} a_{a}^{n-1}-\lambda & a_{2} \alpha_{1} a_{a}^{n-1} & a_{3} \alpha_{1} a_{a}^{n-1} \\
a_{1} \alpha_{2} a_{a}^{n-1} & a_{2} \alpha_{2} a_{a}^{n-1}-\lambda & a_{3} \alpha_{2} a_{a}^{n-1} \\
a_{1} \alpha_{3} a_{a}^{n-1} & a_{2} \alpha_{3} a_{a}^{n-1} & a_{3} \alpha_{3} a_{a}^{n-1}-\lambda
\end{array}\right|=0 .
$$

The coefficients of this cubic are invariants of the two curves.

We shall consider the case where $n=3$, and the point and line cubics degenerate respectively into a 3-line and a 3-point. Let the 3-point be

and the 3-line

$$
a_{\xi} b_{\xi} c_{\xi}=0 \text {, }
$$

$$
\alpha_{x} \beta_{x} \gamma_{x}=0 \text {. }
$$

We shall make use of the following notation:

$$
b_{\beta} c_{\gamma}+b_{\gamma} c_{\beta}=(b c / \beta \gamma),
$$

* Presented to the Society December 28, 1903. Received for publication June 15, 1903.

†.Journal de Mathématiques, ser. 4, vol. 4 (1886), p. 249.

$\ddagger$ HILBERT, loc. cit. 


$$
\begin{aligned}
\sum_{a}(b c / \beta \gamma) a_{i} & =(\beta \gamma)_{i}, \\
\sum_{a}(\beta \gamma)_{i} \alpha_{j} & =\sum_{a}(b c)_{j} a_{i}=B_{j i} .
\end{aligned}
$$

The equation (2) then becomes

$$
\Delta(\lambda)=\left|\begin{array}{ccc}
B_{11}-\lambda & B_{12} & B_{13} \\
B_{21} & B_{22}-\lambda & B_{23} \\
B_{31} & B_{32} & B_{33}-\lambda
\end{array}\right|=0 .
$$

This equation may be written in the form

where

$$
\lambda^{3}+I_{1} \lambda^{2}+I_{2} \lambda+I_{3}=0
$$

$$
\begin{aligned}
I_{1} & =-\sum B_{11} \\
I_{2} & =\sum\left(B_{22} B_{33}-B_{23} B_{32}\right) \\
I_{3} & =-\left|\begin{array}{lll}
B_{11} & B_{12} & B_{13} \\
B_{21} & B_{22} & B_{23} \\
B_{31} & B_{32} & B_{33}
\end{array}\right| \\
& =-|\alpha \beta \gamma| \cdot|a b c| \cdot\left|\begin{array}{lll}
(b c / \beta \gamma) & (b c / \gamma \alpha) & (b c / \alpha \beta) \\
(c a / \beta \gamma) & (c a / \gamma \alpha) & (c a / \alpha \beta) \\
(a b / \beta \gamma) & (a b / \gamma \alpha) & (a b / \alpha \beta)
\end{array}\right|=\Delta D N .
\end{aligned}
$$

\section{$\S 2$. Invariants of the connex.*}

The connex set up by the two triangles is

$$
\sum_{i, j} B_{i j} y_{i} \eta_{j}=0 .
$$

This gives rise to the two collineations

and

$$
Y: \quad y_{i}^{\prime}=\sum_{j} B_{j i} y_{j},
$$

$$
H: \quad \eta_{i}^{\prime}=\sum B_{i j} \eta_{j}
$$

By taking the triangle of reference as the fixed triangle of $Y$, it is at once seen that $Y$ is the inverse of $H$.

*Cl. Clebsch, Géométrie, French translation, vol. 1, p. 311 ff. 
Let the reference triangle be now taken as that of the 3-point. Then

$$
B_{i j}=\sum_{a}\left(\beta_{\kappa} \gamma_{l}+\beta_{l} \gamma_{\kappa}\right) \alpha_{i} \quad(j \neq \kappa \neq l),
$$

and $Y$ sends the point $(1,0,0)$ into $\left(B_{11}, B_{12}, B_{13}\right)$. This point lies on the line $(1,0,0)$ if $B_{11}$ is zero. But

$$
-\frac{1}{3} I_{1}=B_{11}=B_{22}=B_{33}=\sum_{a}\left(\beta_{2} \gamma_{3}+\beta_{3} \gamma_{1}\right) \alpha_{1} \text {. }
$$

If then $I_{1}=0, Y$ sends the 3-point into an inscribed 3-point. Evidently, this is also the condition that $H$ sends the 3-line into a circumscribed 3-line. Since $H$ is the inverse of $Y$, it follows that $Y$ and $H$ respectively send the 3-point and 3-line into inscribed and circumscribed triangles.* A collineation whose first invariant vanishes may be said to be normal.

If $I_{2}=0$, the sum of the minors of the elements of the principal diagonal in the determinant of the connex is zero. The collineations $Y$ and $H$ may then be said to be sub-normal. They have respectively the property of sending certain triangles into circumscribed and inscribed triangles. It is, however, easy to see that neither the 3-point nor the 3-line is such a triangle.

If $I_{3}=0$, the determinant of the connex is zero. In this case zero is a root of (4), and this value of $\lambda$, instead of giving a proper fixed point of $Y$, will give a point $y_{0}$ which $Y$ explodes; i. e., which $Y$ sends anywhere. Similarly, there is a line $\eta_{0}$ which $H$ explodes. In general, $y_{0}$ and $\eta_{0}$ are not incident point and line, and hence may be taken as opposite point and line of the reference triangle. Then

$$
B_{i 1}=B_{1 i}=0 \quad(i=1,2,3) .
$$

The collineation $Y$ sends all points on a line through $y_{0}$ into the same point on $\eta_{0}$. Among these lines there are two all points of which will be sent into their intersections with $\eta_{0}$. These intersections are then the proper fixed points of $Y$; and the lines joining them to $y_{0}$ are the proper fixed lines of $H$. Upon taking the two fixed points of $Y$ as the remaining reference points, the collineations become

$$
\begin{array}{llll}
Y: & y_{1}^{\prime}=0, & y_{2}^{\prime}=B_{22} y_{2}, & y_{3}^{\prime}=B_{33} y_{3}, \\
H: & \eta_{1}^{\prime}=0, & \eta_{2}^{\prime}=B_{22} \eta_{2}, & \eta_{3}^{\prime}=B_{33} \eta_{3} .
\end{array}
$$

Evidently then, if $Y$ sends $y$ into $y^{\prime}, H$ sends any line through $y^{\prime}$ into the join of $y$ and $y_{0}$. Since $I_{3}=\Delta D N$, the collineations may be brought into these forms when the 3-point or 3-line degenerates. It may be shown that, if $\Delta=0, y_{0}$ is the point in which $\alpha, \beta$ and $\gamma$ meet; and, if $D=0$, that $y_{0}$ is the line in which $a, b$ and $c$ lie.

* Cf. PaSch, Matbematische Annalen, vol. 23 (1884), p. 426. 
The invariants of the connex yield then the following invariants of the triangles :

$$
I_{1}(3,3), \quad I_{2}(6,6), \quad N(6,6), \quad \Delta(0,3), \quad D(3,0),
$$

where the numbers in the parentheses indicate the collective degrees in the Roman and Greek letters respectively.

From these five invariants can be built three absolute invariants,

$$
\frac{N}{I_{2}}, \quad \frac{I_{1}}{\Delta D}, \quad \frac{I_{1}^{2}}{I_{2}}
$$

Now two triangles have $12-8=4$ absolute invariants. Thus. it appears that (as we should expect) all invariants of the triangles are not obtainable by a consideration of the connex alone.

\section{§3. Special forms taken by the fundamental invariants.}

In order to determine the invariants of the 3-point and 3-line it will be found to be convenient to know the forms that $I_{1}, I_{2}, \Delta D$ and $N$ assume when the 3 -line is taken as the reference triangle and the points $b$ and $c$ of the 3-point as the absolute.

We have for this special case, by direct substitution and reduction,

$$
\begin{aligned}
I_{1} & =12 \sum c_{1} a_{1}, \\
I_{2} & =16 \sum\left(2 c_{1}^{2}-1\right) a_{1}^{2}+2\left(2-c_{2} c_{3}\right) a_{2} a_{3}, \\
\Delta D & =4 i \sum a_{1}, \\
N & =16 i \sum c_{1} a_{1}^{2}-\left(c_{2}+c_{3}\right) a_{2} a_{3}, \\
\Delta D I_{1} & =48 i \sum c_{1} a_{1}^{2}+\left(c_{2}+c_{3}\right) a_{2} a_{3},
\end{aligned}
$$

where the $c$ 's are the cotangents of the interior angles of the reference triangle.

\section{§4. The interchange of the 3-point and 3-line.}

If the vanishing of some invariant $I$ be found to express the condition that the 3-point and 3-line have a certain projective property, then the vanishing of the invariant formed by substituting in $I$, for each Roman letter the minor in $\Delta$ of the corresponding Greek letter, and for each Greek letter the minor in $D$ of the corresponding Roman letter, must express the condition that the meets of the 3-line and the joins of the 3-point have the same projective property. The algebra involved in making this substitution being extremely long, we shall prove one formula only, and in the others merely give the results obtained. 
By definition

and

$$
I_{2}=\sum_{1}^{3} B_{22} B_{33}-B_{23} B_{32}
$$

Then

$$
B_{i j}=\sum_{a} \sum_{a}(b c / \beta \gamma) a_{j} \alpha_{i}
$$

$$
\begin{aligned}
& B_{22} B_{33}=\left[\sum_{a} \sum_{a}(b c / \beta \gamma) a_{2} \alpha_{2}\right] \cdot {\left[\sum_{a} \sum_{a}(b c / \beta \gamma) a_{3} \alpha_{3}\right] } \\
&=\sum_{a} \sum_{a}\left[(b c / \beta \gamma)^{2} a_{2} \alpha_{2} a_{3} \alpha_{3}+(b c / \gamma \alpha)(b c / \alpha \beta)\left\{a_{2} \beta_{2} a_{3} \gamma_{3}+a_{2} \gamma_{2} a_{3} \beta_{3}\right\}\right. \\
&+(c a / \beta \gamma)(a b / \beta \gamma)\left\{b_{2} \alpha_{2} c_{3} \alpha_{3}+c_{2} \alpha_{2} b_{3} \alpha_{3}\right\} \\
&+(c a / \gamma \alpha)(a b / \alpha \beta)\left\{b_{2} \beta_{2} c_{3} \gamma_{3}+c_{2} \gamma_{2} b_{3} \beta_{3}\right\} \\
&\left.+(c a / \alpha \beta)(a b / \gamma \alpha)\left\{b_{2} \gamma_{2} c_{3} \beta_{3}+b_{3} \gamma_{3} c_{2} \beta_{2}\right\}\right] .
\end{aligned}
$$

Hence,

$$
\begin{aligned}
\sum_{1}^{3} B_{22} B_{33}=\sum_{a} \sum_{a}[( & b c / \gamma \alpha)(b c / \alpha \beta) a_{\beta} a_{\gamma}+(c a / \beta \gamma)(a b / \beta \gamma) b_{a} c_{\alpha} \\
& +(c a / \gamma \alpha)(a b / \alpha \beta) b_{\beta} c_{\gamma}+(c a / \alpha \beta)(a b / \gamma \alpha) b_{\gamma} c_{\beta} \\
& +\sum_{1}^{3}\left\{(b c / \beta \gamma)^{2} a_{2} \alpha_{2} a_{3} \alpha_{3}-(b c / \gamma \alpha)(b c / \alpha \beta) a_{1}^{2} \beta_{1} \gamma_{1}\right. \\
& -(c a / \beta \gamma)(a b / \beta \gamma) b_{1} c_{1} \alpha_{1}^{2}-(c a / \gamma \alpha)(a b / \alpha \beta) b_{1} c_{1} \beta_{1} \gamma_{1} \\
& \left.\left.-(c a / \alpha \beta)(a b / \gamma \alpha) b_{1} c_{1} \beta_{1} \gamma_{1}\right\}\right] .
\end{aligned}
$$

Similarly, we have

$$
\begin{aligned}
\sum_{1}^{3} B_{23} B_{32}=\sum_{a} \sum_{a}\left[(b c / \gamma \alpha)(b c / \alpha \beta) a_{\beta} a_{\gamma}+(c a / \beta \gamma)(a b / \beta \gamma) b_{a} c_{\alpha}\right. \\
+(c a / \gamma \alpha)(a b / \alpha \beta) b_{\gamma} c_{\beta}+(c a / \alpha \beta)(a b / \gamma \alpha) b_{\beta} c_{\gamma} \\
+\sum_{1}^{3}\left\{(b c / \beta \gamma)^{2} a_{2} \alpha_{2} a_{3} \alpha_{3}-(b c / \gamma \alpha)(b c / \alpha \beta) a_{1}^{2} \beta_{1} \gamma_{1}\right. \\
-(c a / \beta \gamma)(a b / \beta \gamma) b_{1} c_{1} \alpha_{1}^{2}-(c a / \gamma \alpha)(a b / \alpha \beta) b_{1} c_{1} \beta_{1} \gamma_{1} \\
\left.\left.-(c a / \alpha \beta)(a b / \gamma \alpha) b_{1} c_{1} \beta_{1} \gamma_{1}\right\}\right] .
\end{aligned}
$$

We have then

$I_{2}=\sum_{a} \sum_{a} a_{a}\left(b_{\beta} c_{\gamma}-b_{\gamma} c_{\beta}\right)\left[a_{a}\left(b_{\beta} c_{\gamma}-b_{\gamma} c_{\beta}\right)-a_{\beta}\left(b_{\gamma} c_{a}-b_{a} c_{\gamma}\right)-a_{\gamma}\left(b_{a} c_{\beta}-b_{\beta} c_{\alpha}\right)\right]$

\section{Let now,}

Then

$$
\alpha_{1}^{\prime}=b_{2} c_{3}-b_{3} c_{2} \text {, etc. }
$$

$$
a_{a^{\prime}}^{\prime}=b_{\beta} c_{\gamma}-b_{\gamma} c_{\beta} \text {. }
$$


Hence,

$$
\begin{aligned}
\left(b_{\beta} c_{\gamma}-b_{\gamma} c_{\beta}\right)^{\prime} & =a_{a}\left[a_{a}\left(b_{\beta} c_{\gamma}-b_{\gamma} c_{\beta}\right)+a_{\beta}\left(b_{\gamma} c_{\alpha}-b_{\alpha} c_{\gamma}\right)+a_{\gamma}\left(b_{a} c_{\beta}-b_{\beta} c_{\alpha}\right)\right] \\
& =\Delta D a_{\alpha} .
\end{aligned}
$$

Substituting these values in the expression for $I_{2}^{\prime}$, we have

$$
\begin{array}{rlr}
I_{2}^{\prime} & =\Delta^{2} D^{2} \sum_{a} \sum_{a} a_{a}\left(b_{\beta} c_{\gamma}-b_{\gamma} c_{\beta}\right)\left[a_{\alpha}\left(b_{\beta} c_{\gamma}-b_{\gamma} c_{\beta}\right)-a_{\beta}\left(b_{\gamma} c_{\alpha}-b_{\alpha} c_{\gamma}\right)\right. \\
& =\Delta^{2} D^{2} I_{2} . & \left.-a_{\gamma}\left(b_{\alpha} c_{\beta}-b_{\beta} c_{\alpha}\right)\right]
\end{array}
$$

By similar calculation we find the following values for the invariants $I_{1}^{\prime}, \Delta^{\prime}, D^{\prime}$ and $N^{\prime}$ :

$$
\left\{\begin{aligned}
I_{1}^{\prime} & =-\frac{1}{2}\left(\Delta D I_{1}-9 N\right), \\
\Delta^{\prime} D^{\prime} & =\Delta^{2} D^{2}, \\
I_{2}^{\prime} & =\Delta^{2} D^{2} I_{2}, \\
N^{\prime} & =\frac{\Delta^{2} D^{2}}{6}\left(\Delta D I_{1}+3 N\right) .
\end{aligned}\right.
$$

\section{§ 5. Invariant relations of the 3-point and 3-line.}

We are now in a position to express the conditions for certain invariant relations of the 3-point and 3-line.

I. The 3-point degenerates if $D=0$.

II. The 3-line degenerates if $\Delta=0$.

III. Let the 3.point be apolar to the 3.line.

Then $a_{\xi} b_{\xi} c_{\xi}$ acting on $\alpha_{x} \beta_{x} \gamma_{x}$ must be zero. That is,

$$
I_{1}=3 \sum_{a}(b c / \beta \gamma) a_{a}=0 .
$$

The required condition is then $I_{1}=0$.

IV. Let the joins of the 3-point be apolar to the meets of the 3-line.

Then the lines $c a$ and $a b$ are apolar to the polar conic of $b c$ as to the meets of the 3-line. That is, if $b$ and $c$ are the absolute $I, J$ and the 3-line the triangle of reference, the lines $a b$ and $a c$ are apolar to the maximum inscribed ellipse, $\sum \xi_{2} \xi_{3}=0$. In other words, the tangents from $a$ to this conic are perpendicular, and hence $a$ lies on the director circle.

If we define the intermediate of two line conics as the locus of points, $a$, such that the lines of the two conics through them form harmonic pairs, the director circle is the intermediate of the ellipse and the absolute. The intermediate * of two conics, $u_{\xi}^{2}=0$ and $v_{\xi}^{2}=0$, is

\footnotetext{
* Salmon, Conic Sections, tenth ed., pp. 306-7.
} 


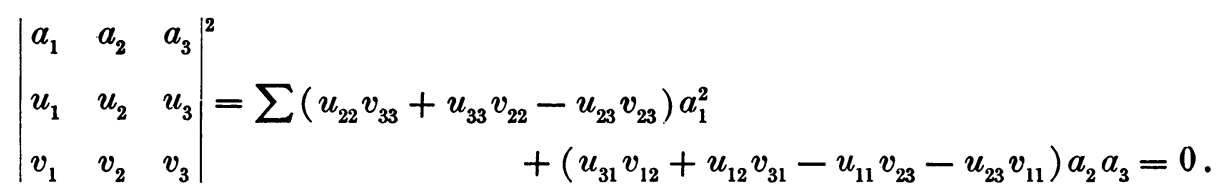

If then

$$
u_{\xi}^{2} \equiv \sum \xi_{2} \xi_{3} \quad \text { and } \quad v_{\xi}^{2} \equiv \sum\left(c_{2}+c_{3}\right) \xi_{1}^{2}-2 c_{1} \xi_{2} \xi_{3},
$$

the director circle becoines

$$
\sum c_{1} a_{1}^{2}-2\left(c_{2}+c_{3}\right) a_{2} a_{3}=0 .
$$

From the equations (5) we have

$$
\Delta D I_{1}-9 N \equiv-96 i \sum c_{1} a_{1}^{2}-2\left(c_{2}+c_{3}\right) a_{2} a_{3} .
$$

The required condition is then

$$
\Delta D I_{1}-9 N=0 \text {. }
$$

V. Let there be a point conic circumscribed to 3-point and apolar to the 3line.

In the special case of $\S 3$, the conic becomes the apolar circle, whose equation is

But, from (5), we have

$$
\sum c_{1} a_{1}^{2}=0 \text {. }
$$

$$
\Delta D I_{1}+3 N=96 i \sum c_{1} a_{1}^{2} .
$$

The required condition is then

$$
\Delta D I_{1}+3 N=0 .
$$

VI. Let there be a line conic touching the joins of the 3-point and apolar to the 3-line.

The equations (6) applied to the invariant $V$ give the required invariant, to within powers of $\Delta$ and $D$, in the form $N=0$. To determine the proper degree we proceed as follows. Let accented Greek letters denote the minors of the corresponding letters in $\Delta$.

Any line conic apolar to the 3 -line is given by

$$
\sum m_{1}\left(\alpha_{\xi}^{\prime}\right)^{2}=0,
$$

and any line conic inscribed to the 3-point by

$$
\sum m_{4} b_{\xi} c_{\xi}=0 \text {. }
$$

We wish then these two conics to be identical. Upon equating the coefficients, we have six homogeneous equations in the six $m$ 's. They are consistent 
if their determinant vanishes. This determinant is then the invariant we wish to find. It is evidently of the sixth degree in the Roman letters and of the twelfth in the Greek letters. The required invariant is then

$$
\Delta^{2} N=0 \text {. }
$$

This invariant may also be easily found directly. In fact, in the special case of $\S 3$, we have only to make $a$ the focus of an apolar parabola. The joins of the middle points of a triangle are lines of all parabolas apolar to the triangle. But a circle, circumscribing a triangle formed by three tangents to a parabola, passes through the focus.*

Then $a$ must lie on the circle passing through the middle points of the sides of the 3 -line; i. e., on the Feuerbach circle, whose equation is

$$
N=16 i \sum c_{1} a_{1}^{2}-\left(c_{2}+c_{3}\right) a_{2} a_{3}=0 .
$$

The remaining factor in the invariant is found as before.

Since the invariant contains the factor $\Delta$, it can only put two conditions on a conic to make it apolar to a degenerate 3 -line. This may also be easily shown directly.

VII. Let there be a conic circumscribed to the 3-point and to the 3-line.

In the case of $\S 3$, the conic becomes the circumcircle, whose equation is

From (5) we have

$$
\sum\left(c_{2}+c_{3}\right) a_{2} a_{3}=0 .
$$

$$
\Delta D I_{1}-3 N=96 i \sum\left(c_{2}+c_{3}\right) a_{2} a_{3} .
$$

This invariant is then a factor in the required condition, which an investigation of the degree shows to be

$$
\Delta^{2}\left(\Delta D I_{1}-3 N\right)=0 .
$$

VIII. Let there be a conic inscribed to the 3-line and to the 3-point.

The required condition is found by an interchange of the Greek and Roman letters in the preceding condition. It is then

$$
D^{2}\left(\Delta D I_{1}-3 N\right)=0 .
$$

If neither the 3-point nor the 3-line degenerates, the conditions VII and VIII are the same. We have then an immediate proof of the well-known theorem $\dagger$ that the vertices of two triangles, circumscribed to a conic, lie on a conic.

IX. Lat there be a line conic apolar to the 3-line and to the 3-point.

The line conic apolar to the 3-point is of the form

* SALMUN. 1 om, sictions, tenth ed., p 207.

†SALMon, Conir Sictions. tenth ed., pp. 320 and 343. 


$$
\sum m_{1}\left(a_{\xi}\right)^{2}=0,
$$

and a line conic apolar to the 3 -line is of the form

These conics are identical if

$$
\sum m_{4}\left(\alpha_{\xi}^{\prime}\right)^{2}=0 \text {. }
$$

$$
\sum m_{1} a_{i} a_{j}=\sum m_{4} \alpha_{i}^{\prime} \alpha_{j}^{\prime} .
$$

The condition for consistency of these six equations is the vanishing of the same determinant as that found in investigating the degree of the invariant VII.

The required condition is then

$$
\Delta^{2}\left(\Delta D I_{1}-3 N\right)=0 .
$$

X. Let there be a point conic apolar to the 3-line and to the 3-point.

The required invariant condition is found from the preceding by an interchange of the Greek and Roman letters. It is then

$$
D^{2}\left(\Delta D I_{1}-3 N\right)=0 .
$$

It is obvious that the substitution (6) applied to IX and $\mathrm{X}$ must merely reproduce these conditions. This is at once seen to be the case.

$\mathrm{XI}$. Let there be a point $y$ such that its polar conic as to the 3-line is apolar to the 3-point.

Referring to $\S 2$, we see that $y$ is exploded by the collineation $Y$. The condition that there may be such a point is then

$$
I_{3}=0 \text {. }
$$

XII. Let there be a point $y$ such that its polar conic as to the joints of the 3-point is apolar to the meets of the 3-line.

By applying the substitution (6) to the preceding invariant, and investigating the degree, we find the required condition in the form

$$
\Delta^{2} D^{2}\left(\Delta D I_{1}+3 N\right)=0 .
$$

XIII. Let the three polar lines, as to the 3-line, of the points of the 3-point, taken two at a time, meet in a point.

The polar line of $b_{\xi} c_{\xi}$ as to the 3-line is

$$
\sum_{a}(b c / \beta \gamma) \alpha_{x} \equiv \sum_{1,2,3}(b c)_{1} x_{1}=0 .
$$

These three lines meet in a point if

$$
\left|(b c)_{1},(c a)_{2},(a b)_{3}\right| \equiv \Delta N=0 .
$$

This then gives a simple projective definition of the Feuerbach conic of two points and a triangle, where we mean by the Feuerbach conic that conic which 
will project into the Feuerbach circle when the two points are projected into the absolute.

XIV. Let there be a collineation, having the 3-line as its fixed triangle, which sends the 3-point into an inscribed 3-point.

If the 3 -line be the triangle of reference, the collineation is of the form

$$
x_{i}^{\prime}=k_{i} x_{i}
$$

This collineation sends $a$ into a point on the join of $b$ and $c$ if

$$
\sum a_{1} A_{1} k_{1}=\left|\begin{array}{rrr}
a_{1} k_{1} & a_{2} k_{2} & a_{3} k_{3} \\
b_{1} & b_{2} & b_{3} \\
c_{1} & c_{2} & c_{3}
\end{array}\right|=0 .
$$

Similarly, the conditions that $b$ and $c$ are sent into points on the joins of $c$ and $a$, and $a$ and $b$ respectively are

$$
\sum b_{1} B_{1} k_{1}=0 \quad \text { and } \quad \sum c_{1} C_{1} k_{1}=0 .
$$

Adding these three equations, we have, as the condition upon the collineation itself,

$$
k_{1}+k_{2}+k_{3}=0 \text {. }
$$

Elimination of the $k$ 's gives, as the condition the 3-point must satisfy,

$$
\begin{aligned}
& \left|\begin{array}{lll}
a_{1} A_{1} & a_{2} A_{2} & a_{3} A_{3} \\
b_{1} B_{1} & b_{2} B_{2} & b_{3} B_{3} \\
c_{1} C_{1} & c_{2} C_{2} & c_{3} C_{3}
\end{array}\right| \\
& =\sum a_{1}\left(b_{2} c_{3}-b_{3} c_{2}\right)\left[b_{2} c_{3}\left(c_{3} a_{1}-c_{1} a_{3}\right)\left(a_{1} b_{2}-a_{2} b_{1}\right)\right. \text {. } \\
& \left.-b_{3} c_{2}\left(c_{1} a_{2}-c_{2} a_{1}\right)\left(a_{3} b_{1}-a_{1} b_{3}\right)\right] \\
& =\sum a_{1}\left(b_{2} c_{3}-b_{3} c_{2}\right)\left[a_{1}^{2}\left(b_{2}^{2} c_{3}^{2}-b_{3}^{2} c_{2}^{2}\right)+a_{2} a_{3} b_{1} c_{1}\left(b_{2} c_{3}-b_{3} c_{2}\right)\right. \\
& \left.-a_{3} a_{1}\left(b_{2}^{2} c_{3} c_{1}-b_{3} b_{1} c_{2}^{2}\right)-a_{1} a_{2}\left(b_{1} b_{2} c_{3}^{2}-b_{3}^{2} c_{1} c_{2}\right)\right]=0 \text {. }
\end{aligned}
$$

If now $b$ and $c$ are sent into the absolute, this equation becomes

or

$$
\sum a_{1}\left[2 c_{1} a_{1}^{2}+\left(c_{3}-c_{1}\right) a_{3} a_{1}+\left(c_{2}-c_{2}\right) a_{1} a_{2}-\left(c_{2}+c_{3}\right) a_{2} a_{3}\right]=0,
$$

$$
\left(a_{1}+a_{2}+a_{3}\right) \sum 2 c_{1} a_{1}^{2}-\left(c_{2}+c_{3}\right) a_{2} a_{3}=0 .
$$

But from the equations (5) we have

$$
\Delta D I_{1}+9 N=96 i \sum 2 c_{1} a_{1}^{2}-\left(c_{2}+c_{3}\right) a_{2} a_{3} .
$$


Hence, the required condition is

The conic

$$
D\left(\Delta D I_{1}+9 N\right)=0 .
$$

$$
\sum 2 c_{1} a_{1}^{2}-\left(c_{2}+c_{3}\right) a_{2} a_{3}=0
$$

belongs to the pencil of circles determined by the apolar- and circumcircles. Also, it evidently passes through the centroid $(1,1,1)$ and orthocentre $\left(1 / c_{1}, 1 / c_{2}, 1 / c_{3}\right)$, and it may be shown that the polar of the middle point of the line joining these two points is the line at infinity. Then, if the 3-point does not degenerate, $a$ must lie on the ortho-centroidal circle of the 3-line: $\mathrm{i}$. e., on the circle having the join of the orthocentre and centroid as a diameter.

$\mathrm{XV}$. Let there be a collineation, having the 3-point as fixed triangle, which sends the 3-line into an inscribed 3-line.

The required condition may evidently be found by applying the substitution (6) to the equation

This gives

$$
D\left(\Delta D I_{1}+9 N\right)=0 .
$$

$$
\Delta\left(\Delta D I_{1}+9 N\right)=0 \text {. }
$$

The condition is then, upon neglecting the cases of degenerate 3-line and 3point, the same as that of XIV. Hence we have the theorem:

If there exists a collineation, having fixed points at $a, b$ and $c$, which sends each of the points $A, B$ and $C$ into a point on the join of the other two, then there also exists a collineation, with fixed points at $A, B$ and $C$, which sends each of the points $a, b$ and $c$ into a point on the join of the other two.*

The vanishing of the invariant $\Delta D I_{1}+9 N$ then expresses the fact that there is a mutual relation between the two triangles. The condition that the vanishing of an invariant express such a relation is that the substitution (6), applied to that invariant, shall merely reproduce it.

We have

$$
\Delta^{\prime} D^{\prime} I_{1}^{\prime}+\lambda N^{\prime}=\frac{\Delta^{2} D^{2}}{6}\left[(\lambda-3) \Delta D I_{1}+3(9+\lambda) N\right] .
$$

If this is to be identical to

$$
k \Delta^{2} D^{2}\left[\Delta D I_{1}+\lambda N\right],
$$

we must have $\lambda$ equal to -3 or 9 . Then the only invariants of the pencil the vanishing of which express mutual relations are

$$
\Delta D I_{1}+9 N \text { and } \Delta D I_{1}-3 N .
$$

The former we have just considered, and the latter is the condition that there be a conic circumscribed to the 3-line and to the 3-point. From the substitu-

* F. Morley, Projective Coördinates, Transactions of the American Mathematical Society, vol. 4 (1903), p. 292. 
tion (6), we also see that the vanishing of the invariant $I_{2}$ expresses a mutual relation between the two triangles. An adequate interpretation of this relation has not however presented itself.

The results of $\S 5$ may be tabulated as follows:

The 3-point degenerates, if

$$
D=0 \text {. }
$$

The 3-point is apolar to the 3-line, if

$$
I_{1}=0 \text {. }
$$

The meets of the 3-line are apolar to the joins of the 3-point, if

$$
\Delta D I_{1}-9 N=0 \text {. }
$$

A conic, circumscribed to the 3-point and apolar to the 3 -line, exists, if

$$
\Delta D I_{1}+3 N=0 \text {. }
$$

A point conic, apolar to both the 3-point and 3-line, exists, if

$$
D^{2}\left(\Delta D I_{2}-3 N\right)=0 \text {. }
$$

A conic, circumscribed to both the 3-point and 3-line, exists if

$$
\Delta D I_{1}-3 N=0 .
$$

There exists a point, whose polar conic as to the 3-line is apolar to the 3-point, if

$$
\Delta D N=0 \text {. }
$$

The three polar lines, as to the 3-line, of the points of the 3-point, taken two at a time, meet in a point, if

$$
\Delta N=0 \text {. }
$$

There exists a collineation, having the 3-point as fixed triangle, which sends each meet of the 3-line into a point on the opposite line, if

$$
\Delta D I_{1}+9 N=0 .
$$

The 3.line degenerates, if

$$
\Delta=0 \text {. }
$$

The 3-line is apolar to the 3-point, if

$$
I_{1}=0 \text {. }
$$

The joins of the 3-point are apolar to the meets of the 3-line, if

$$
\Delta D I_{1}-9 N=0 .
$$

A conic, inscribed to the 3 -line and apolar to the 3-point, exists, if

$$
\Delta D I_{1}+3 N=0 \text {. }
$$

A line conic, apolar to both the 3-line and 3-point, exists, if

$$
\Delta^{2}\left(\Delta D I_{2}-3 N\right)=0 .
$$

A conic, inscribed to both 3-line and 3-point, exists if

$$
\Delta D I_{1}-3 N=0 .
$$

There exists a line, whose polar conic as to the 3-point is apolar to the 3-line, if

$$
\Delta D N=0 \text {. }
$$

The three polar points, as to the 3-point, of the lines of the 3-line, taken two at a time, lie on a line, if

$$
D N=0 \text {. }
$$

There exists a collineation, having the 3-line as fixed triangle, which sends each join of the 3-point into a line through the opposite point, if

$$
\Delta D I_{1}+9 N=0 \text {. }
$$


There exists a collineation, having the meets of the 3 -line as fixed points, which sends each point of the 3-point into a point on the join of the other two, if

$$
\Delta D I_{1}+9 N=0 \text {. }
$$

There exists a collineation, having the joins of the 3-point as fixed lines, which sends each line of the 3 -line into a line through the meet of the other two, if

$$
\Delta D I_{1}+9 N=0 .
$$

$\S 6$. Two triangles apolar in both ways.

If the two invariants, $\Delta D I_{1}-3 N$ and $\Delta D I_{1}+3 N$, simultaneously vanish, the two triangles formed by the 3-point and 3-line are apolar in both ways. But, if $a$ be considered as the variable point, these invariants represent a circumconic and an apolar conic of the 3-line, which meet in four points $I, J, K$, and $L$. These points pair off into two on the line $D$ and two on the line $I_{1}$, the former pair being of course, the points $b$ and $c$, assumed for the moment to be $I$ and $J$.

If then $a$ coincides with either $K$ or $L$, it forms with $I$ and $J$ a triangle apolar in both ways to that of the 3-line. An interchange of the pairs $I, J$ and $K, L$ merely interchanges the lines $D$ and $I_{1}$. Then the triangle formed by any three of the points $I, J, K, L$ is apolar in both ways to that of the 3 -line. That is, the points $I, J, K, L$ form a conjugate 4-point of the 3-line.*

We shall now show that the Feuerbach conic of the 3-line, $I$ and $J$ is identical with that of the 3-line, $K$ and $L$.

Let $a$ be a point on the line $I_{1}$, and let $b$ and $c$ be the absolute. Then the polar of $a$ as to the 3-line is apolar to the absolute. If $a^{(1)}$ denote the point where $\alpha$ cuts the line $I_{1}$, then the polar conic of $a^{(1)}$ as to the 3-line brealss up into the line $\alpha$ and the perpendicular from the opposite vertex. It follows that the polar point of the line $I_{1}$ as to the 3-line is the orthocentre.

The Feuerbach conic of $K, L$ and the 3 -line may be defined as the conic through $K, L$ and the polar points of the join of $K$ and $L$ as to the meets of the 3-line taken two at a time. This corresponds to the fact that the Feuerbach circle passes through the middle points of the sides. But, we have seen that the polars of $I_{1}$ as to the meets of the 3-line are the feet of the perpendiculars from the vertices of the 3-line. Then, since the Feuerbach circle also passes through these points, the two conics have five points in common, and so coincide.

Hence, the Feuerbach conic of a 3-line and any two points of a conjugate 4-point is identical with that of the 3-line and the two other points.

\section{An extension of Majcen's theorem. $\dagger$}

MAJCEN shows that if we project the points of the triangle $A B C$, from an intersection of the circumcircle and apolar circle, upon the apolar circle the tri-

* Caporali, Works, p. 51.

† G. MaJcen, Archiv der Mathematik und Physik, ser. 3, vol. 4 (1903), pp. 94, 95. 
angle so formed $A^{*} B^{*} C^{*}$ will be circumscribed and similar to $A B C$. In this section we shall prove the following extension of this theorem:

There exists a collineation, leaving fixed three points of a conjugate 4-point of a 3-line, which sends the 3-line into a circumscribed, perspectivc 3-line, the center of perspective being the remaining point of the 4-point, and the conic through the fixed points and circumscribed to the new 3-line being apolar to the original 3-line.

We shall make use of the system of circular coördinates. A point of the plane will be defined by two conjugate complex quantities $x$ and $\bar{x}$, the real and imaginary parts of which are respectively the $x$ and $y$ rectangular Cartesian coördinates of the point. In order that $x$ may always define a single point, the convention is made that infinity is a point.

The bilinear substitution

$$
x^{\prime}=\frac{a x+b}{c x+d}
$$

sends circles into circles, provided that a line be considered as a circle through the infinite point. The substitution has, in general, two fixed points. If infinity be one of these, the substitution sends circles and lines into circles and lines, and is orthomorphic. It is then identical with a collineation having fixed points at the absolute.

Let the meets of the 3 -line be the points $a_{1}, a_{2}$, and $a_{3}$, and so take the axes and unit of measure that these points lie on the unit circle, and that the Euler line of the triangle $a$ is the axis of reals. Further, let $s_{i}$ denote the sum of the $a$ 's $i$ at a time. The orthocenter and centroid are then the points $s_{1}$ and $s_{1} / 3$ respectively.

Any substitution, with fixed points at infinity and $K$, is of the form

$$
x^{\prime}=\mu(x-K)+K \text {. }
$$

If this is to send $a_{1}$ into a point on the join of $a_{2}$ and $a_{3}$, we must have

$$
(1+\lambda)\left[\mu\left(a_{1}-K\right)+K\right]=a_{2}+\lambda a_{3}
$$

for a real $\lambda$. The conjugate of this equation is

$$
\frac{a_{2} a_{3}}{a_{1}}(1+\lambda)\left[\bar{\mu}\left(1-a_{1} \bar{K}\right)+a_{1} \bar{K}\right]=a_{3}+\lambda a_{2},
$$

and hence, by addition,

$$
\mu\left(a_{1}-K\right) a_{1}+K a_{1}+a_{2} a_{3} \bar{\mu}\left(1-a_{1} \bar{K}\right)+s_{3} \bar{K}=a_{1}\left(a_{2}+a_{3}\right) .
$$

Similarly we have

$$
\mu\left(a_{2}-K\right) a_{2}+K a_{2}+a_{3} a_{1} \bar{\mu}\left(1-a_{2} \bar{K}\right)+s_{3} \bar{K}=a_{2}\left(a_{3}+a_{1}\right) .
$$


Hence, by subtraction,

$$
\begin{aligned}
& \mu\left(a_{1}+a_{2}-K\right)+K-a_{3} \bar{\mu}=a_{3}, \\
& \mu\left(a_{2}+a_{3}-K\right)+K-a_{1} \bar{\mu}=a_{1} .
\end{aligned}
$$

Eliminating from the last two equations first $k$ and then $\bar{\mu}$, we have

and

$$
\mu+\bar{\mu}+1=0,
$$

$$
K=\frac{\mu s_{1}}{\mu-1} .
$$

Hence the condition upon the collineation is that the real part of $\mu$ is $-\frac{1}{2}$. The condition upon $K$ is that it must run along the ortho-centroidal circle of the triangle $a .^{*}$

Since the intersections of the circum- and apolar circles lie on the ortho-centroidal circle, there exists a substitution, with fixed points at infinity and either of these intersection points, which sends the triangle $a$ into an inscribed triangle. The Feuerbach circle also belongs to this pencil, and therefore the points of intersection may be determined from the circum- and Feuerbach circles.

The substitution

$$
x^{\prime}=-\frac{1}{2}\left(x-s_{1}\right)
$$

sends the points of the triangle each into the middle point of the join of the other two, and therefore sends the circumcircle into the Feuerbach circle. The map-equation of the latter is then

as $t$ runs along the unit circle.

$$
y=-\frac{1}{2}\left(t-s_{1}\right)
$$

If $y$ be also on the circumcircle, $|y|=1$, and hence the points of intersection are found by solving

We have

$$
\left|\frac{t-s_{1}}{2}\right|=|t|=1
$$

Then

$$
\left|\frac{t-s_{1}}{2}\right|=\frac{1}{4}\left(t-s_{1}\right)\left(\frac{1}{t}-s_{1}\right)=1 \text {. }
$$

where

$$
t=\frac{-2-B \pm i A}{2 s_{1}}
$$

$$
B=1-s_{1} \quad \text { and } \quad A=\sqrt{-B(B+8)} \cdot \dagger
$$

The points of intersection are then

\footnotetext{
* That $K$ must run along the ortho-centroidal cirole as $\mu$ runs along the line $-\frac{1}{2}$ maj be deduced from the results of $\S 5, \mathrm{XIV}$, or may be easily shown directly.

$\dagger A$ is real or imaginary according as the points of intersection are real or imaginary.
} 


$$
K=\frac{1}{4 s_{1}}(4-B+i A), \quad L=\frac{1}{4 s_{1}}(4-B-i A) .
$$

If then in the substitution (7) we replace $\mu$ and $K$ by their values found from (8) and (9), we shall have a substitution, $\mathrm{L}$, with fixed points at infinity and $K$, which sends the triangle $a$ into an inscribed triangle $a^{\prime}$.

This substitution is

$$
\mathrm{L}: \quad x^{\prime}=\frac{-B+i A}{2 B}\left(x-s_{1}\right) .
$$

The points $L$ and $a_{1}$ are sent into

and

$$
L^{\prime}=\frac{-B-2+i A}{2 s_{1}}
$$

$$
a_{1}^{\prime}=\frac{-B+i A}{2 B}\left(a_{1}-s_{1}\right) \text {. }
$$

Then the three point $a_{1}, a_{1}^{\prime}$, and $L^{\prime}$ lie on a line if

$$
(1+\lambda) \frac{-B-2+i A}{2 s_{1}}=\frac{-B+i A}{2 B}\left(a_{1}-s_{1}\right)+\lambda a_{1}
$$

for a real $\lambda$. The conjugate of this equation is

Eliminating $a_{1}$, we have

$$
(1+\bar{\lambda}) \frac{-B-2-i A}{2 s_{1}}=\frac{-B-i A}{2 B}\left(\frac{1}{a_{1}}-s_{1}\right)+\frac{\bar{\lambda}}{a_{1}} .
$$

$$
A B(1-B)(\lambda-\bar{\lambda})=0 .
$$

Then $\lambda$ is real unless $B=0,-8$ or 1 .

If $B=0$, the substitution sends all points into infinity.

If $B=-8, s_{1}$ is equal to \pm 3 , and the condition that $a_{1}, a_{1}^{\prime}$, and $L^{\prime}$ lie on a line becomes

$$
\pm(1+\lambda)=-\frac{1}{2} a_{1} \pm \frac{3}{2}+\lambda a_{1},
$$

which is satisfied if $\lambda=1 / 2$.

If $B=1, s_{1}$ is zero, and therefore $L^{\prime}$ is infinite.

Then, in all cases, the points $a_{1}, a_{1}^{\prime}$, and $L^{\prime}$ lie on a line. It follows that the triangles $a$ and $a^{\prime}$ are in perspective, and that the center of perspective is the point $L^{\prime}$. The two triangles are also similar since infinity is a fixed point of the substitution.

The inverse substitution

$$
\mathrm{L}^{-1}: \quad x^{\prime}=\frac{B+i A}{4} x+s_{1},
$$


sends $a$ into a circumscribed, similar and perspective triangle $a^{\prime \prime}$, the center of perspective being the point $L$. Since $\mathrm{L}^{-1}$ sends the circumcenter, $O$, into the orthocenter, $s$, and leaves $K$ fixed, it sends the circumcircle into the apolar circle. That is, the circumcircle of $a^{\prime \prime}$ is the apolar circle of $a$. We have then proved the theorem stated at the beginning of this section.*

JoHNS HOPKINS UNIVERSITY,

May, 1903.

* If the conic through the fixed points and cironmscribed to $a$ be apolar in both ways to that through the fixed points and apolar to $a$, the triangles $a, a^{\prime}$, and $a^{\prime \prime}$ form a closed system. Each is circumscribed to and in perspective with the one following. These triangles form a special case of the $(3,3)_{9}$ configuration $a$. See KANToR, Lber die Configurationen $(3,3)$ mit den Indices 8, 9, Wiener Sitzungsberichte, vol. 84, (1881), p. 918. 